\title{
La protéine C/EBP et l'équilibre entre prolifération et différenciation
}

La protéine C/EBP (CAAT/enhancer binding protein) est synthétisée dans le foie adulte différencié et dans nombre d'autres tissus à des stades de différenciation terminale, notamment les adipocytes, l'intestin, le poumon, etc. [1]. Elle se lie à des séquences d'ADN présentes dans divers enhancers et dans les promoteurs de gènes codant pour des protéines spécifiques de la différenciation hépatique et adipocytaire, notamment impliquées dans le contrôle des métabolismes ( $m / s n^{\circ} 5$, vol. 6, p. 489) [2, 3]. On trouve ainsi des sites de fixation pour $\mathrm{C} / \mathrm{EBP}$ dans les promoteurs des gènes de l'albumine, d'enzymes glycolytiques comme l'aldolase B, ou gluconéogéniques comme la phosphoénol-pyruvate carboxykinase, d'enzymes de la lipogenèse, etc. [2, 3]. $\mathrm{C} / \mathrm{EBP}$ est un activateur transcriptionnel important des gènes énumérés ci-dessus, appartenant à une famille d'activateurs reconnaissant les mêmes séquences cibles d'ADN, ou des séquences homologues. Deux autres membres de cette famille ont été bien étudiés : DBP ( $D$ element binding protein) [4] et LAP (liver-specific activating protein) [5], ce dernier étant probablement identique aux facteurs NF-IL6, IL-6DBP et IgEBP1 décrits par d'autres équipes $[6,7,8]$. Au point de vue structural, C/EBP se fixe à l'ADN par les régions basiques des deux sous-unités d'un dimère lié par une région dite de fermeture à glissière de leucines (leucine-zipper) [9]. L'essentiel des connaissances accumulées sur C/EBP l'a été par l'équipe de Steven L. McKnight (Baltimore, MD, USA) qui vient de ce facteur était un bloqueur de prolifération et un auxiliaire important de différenciation [10]. Lorsqu'un vecteur d'expression commandant la synthèse de C/EBP est introduit dans des cellules en division, qu'il s'agisse de cellules hépatomateuses, de fibroblastes ou d'adipoblastes, elles cessent de se diviser, si bien qu'il n'est pas possible de cloner des cellules synthétisant activement $\mathrm{C} / \mathrm{EBP}$ : synthèse de $\mathrm{C} / \mathrm{EBP}$ et prolifération semblent antinomiques. R.M. Umek et al. ont donc construit par génie génétique un gène codant pour une protéine hybride constituée de C/EBP et, à son extrémité carboxyterminale, du site de fixation de l'hormone du récepteur des œstrogènes [10].

Dans une telle protéine chimère, les fonctions de C/EBP sont masquées en l'absence d'œstrogènes. Des clones d'adipoblastes ont donc été obtenus, qui synthétisent la protéine $\mathrm{C} / \mathrm{EBP}$ hybride, inactive en l'absence d'œstrogènes. L'addition d'œstrogènes au milieu de culture active $\mathrm{C} / \mathrm{EBP}$ et aboutit à l'arrêt des divisions cellulaires, qui reprennent néanmoins dès que l'hormone est ôtée [10]. Cette activation de C/EBP et cet arrêt de prolifération ne suffisent cependant pas à provoquer la différenciation terminale des adipoblastes en adipocytes, différant en cela des protéines de la famille MyoD - qui, elles aussi, bloquent la division cellulaire, mais sont capables d'induire la différenciation terminale en myotubes [11]. Il faut, pour que la différenciation en adipocytes s'effectue, ajouter toute une série d'hormones, insuline et glucocorticoïdes, notamment. Ce résultat pouvait être attendu, puisque $\mathrm{C} / \mathrm{EBP}$ n'a pas la très grande spécificité musculaire de MyoD1 et doit donc intervenir dans d'autres phénomènes de différenciation que ceux concernant la lignée adipocytaire. Elle doit donc se comporter comme un auxiliaire de différenciation, en coopération avec des agents spécifiques différents selon les types cellulaires. Cet auxiliaire de différenciation est aussi un inhibiteur de prolifération... comme, d'une ccrtaine manière, MyoD1, ct peut donc être considéré comme un " antioncogène". Les prédictions concernant l'effet d'une mutation somatique de C/EBP sont qu'elle pourrait induire une hyperplasie, peut-être un cancer en coopération avec d'autres oncogènes, associés à un blocage de différenciation : de telles caractéristiques sont fréquentes dans les proliférations bénignes ou malignes et il sera particulièrement intéressant de déterminer s'il existe des altérations du gène $C / E B P$ dans certaines tumeurs ou hyperplasies. Il existe d'ailleurs des antécédents à l'apparition de néoplasies à la suite d'altération de gènes de différenciation : le produit du proto-oncogène c-erb $A$, récepteur des hormones thyroïdiennes, qui, entre autres effets, interviendrait normalement dans la différenciation érythroïde, serait compétitivement déplacé de ses cibles sur l'ADN par le produit de son équivalent viral v-erbA, ce qui induirait, en coopération avec c-erbB, une prolifération de précurseurs érythoblastiques $\left(\mathrm{m} / \mathrm{s} n^{\circ} 8\right.$, vol. 5, p. 601). Le récepteur de l'acide rétinoïque, jouant lui aussi un très grand rôle dans le déve- 
loppement embryonnaire et la morphogenèse, est altéré dans les leucémies à promyélocytes $\left(\mathrm{m} / \mathrm{s} \quad n^{\circ} 8\right.$, vol. 6, p. 814). Ces résultats illustrent les multiples interactions entre la prolifération, la différenciation et l'oncogenèse, les gènes initialement caractérisés par leur intervention à l'un de ces niveaux ayant toutes les chances d'influer aussi sur les deux autres.

A. K.

1. Birkenmeier EH, Gwynn B, Howard S, el al. Tissue-specific expression, developmental regulation, and genetic mapping of the gene encoding CCAAT/enhancer binding protein. Genes Dev 1989 ; 3 : 1146-56

2. Friedman $\mathrm{AD}$, Landschulz $\mathrm{WH}$
McKnight SL. CCAAT/enhancer binding protein activates the promoter of the serum albumin gene in cultured hepatoma cells. Genes Dev 1989; 3 : 1314-22.

3. Christy RJ, Yang VW, Ntambi JM, et al. Differentiation-induced gene expression in 3T3-L1 pre-adipocytes : CCAAT/enhancer binding protein interacts with and activates the promoter of two adipocytes-specific genes. Genes Dev 1989; 3: 1323-35

4. Mueller C, Maire P, Schibler U. DBP, a liver-enriched transcriptional activator is expressed late in ontogeny and its tissue specificity is determined post-transcriptionally. Cell 1990 61 : 279-91.

5. Descombes P, Chojkier M, Lichsteiner S, Falvey E, Schibler U. LAP, a novel member of the C/EBP gene family, encodes a liverenriched transcriptional activator protein. Genes Dev 1990 ; 4 : 1541-51.

6. Akira S, Isshiki $\mathrm{H}$, Sugita $\mathrm{T}$, et al. A nuclear factor for IL-6 expression (NF-IL-6) is a member of a C/EBP family. Embo J 1990 ; $1897-990$

7. Poli V, Mancini FP, Cortese R. IL-6DBP, a nuclear protein involved in interleukin 6 signal transduction, defines a new family of leucine-zipper proteins related to C/EBP. Cell $1990 ; 63$ : 643-53.

8. Roman C, Platero S, Shuman J, Calame K. Ig/EBP1: a ubiquitously expressed immunoglobulin enhancer binding protein that is similar to $\mathrm{C} / \mathrm{EBP}$ and heterodimerizes with C/EBP. Genes Dev 1990; 4 : 1404-15. 9. Landschulz WH, Johnson PF, McKnight SL. The DNA binding domain of the rat liver nuclear protein C/EBP is bipartite. Science 1989 ; 243 : 1681-8.

10 Umek RM, Friedman AD, McKnight SL. CCAAT/enhancer binding protein : a component of differentiation switch. Science 1991 ; 251: 288-92.

11. Alonso S. Des facteurs de régulation spécifiques de la myogenèse. médecine/sciences 1990 ; $6 \cdot 635-45$

\section{BRÈVES}

Un gènc pour le syndrome de Turner : une piste à suivre. Le syndrome de Turner associc une petite taille, une dysgénésie gonadique ainsi qu'un ensemble dysmorphique et malformatif complexe. La formule caryotypique génćralement retrouvée dans ce syndrome est (45, $\mathrm{XO})$ ou monosomic X. FergusonSmith avait, dès 1965, émis l'hypothèse que le syndrome de Turner ćtait dû à la perte d'un ou de plusieurs gènes commun(s) à $\mathrm{X}$ et à $\mathrm{Y}$. Un ou de tels gènes se devaient alors d'échapper à l'inactivation d'X. Par la suite, différentes observations de phénotype turnérien à caryotype (46, $X Y)$ avec remaniement délétionnel complexe du Y ont permis de circonscrire sur ce chromosome une région minimale de 90 kilobases susceptible de contenir un tel gène. C'est en clonant cette région que l'équipe de D. Page vient de mettre en évidence un gène susceptible de répondre à ces différents critères. L'analyse de la séquence codante révèle une protéine de 263 acides $\mathrm{m} / \mathrm{s} n^{\circ} 3$, vol. 7 , mars 91 aminés dont l'homologic avec la protéine ribosomique S4 (une des protéines de la petite sous-unitć ribosomique) du rat atteint $93 \%$. Une isoforme de cette protéine ribosomale du Y (RPS4Y) est également portée par le chromosome X (RPS4X), avec, cependant, une divergence de la séquence codante sur 19 acides aminés. L'expression, analysée sur le plan transcriptionnel, paraît ubiquitaire (un transcrit de $1 \mathrm{~kb}$ est détecté dans toutes les lignées et tous les tissus examinés) et, surtout, elle est indépendante du statut actif ou inactif du X qui porte ce gène. Si les critères attendus pour un tel gènc, à savoir sa localisation sur l'Y et le fait qu'il échappe à l'inactivation, en font un bon candidat, en revanche sa localisation sur le bras long du chromosome X en Xq13.1 est beaucoup plus surprenante. En effet, les gènes échappant à l'inactivation décrits jusqu'ici étaient tous localisés sur l'extrémité du bras court du chromosome X. La découverte de deux gènes codant pour une protéine ribo- somique, situés l'un sur le chromosome $Y$ et l'autre sur l'X, est en soi originale, mais leur intervention dans le syndrome de Turner reste encore à démontrer. En effet, la monosomie de RPS4 n'est sans doute pas, à elle seule, responsable de l'ensemble du phénotype clinique, celui-ci ćtant parfois rencontré en cas de délétion limitée au bras court du chromosome X. Beaucoup de questions sont encore non résolues. Il reste notamment à élucider les mécanismes qui président à l'échappement de certains zygotes porteurs d'un caryotype (45, XO) à la létalité, largement majoritaire dans ce type d'anomalie chromosomique. De même, la différence de séquence entre l'ADNc porté par l'X et celui porté par l'Y est-elle responsable d'une différence fonctionnelle des produits protéiques pour lesquels ils codent? Affaire à suivre...

[1. Ferguson-Smith MA, et al. I Med Genet 1965 ; 2, 142-55.]

[2. Fisher EM, et al. Cell $1990 ; 63$ 1205-8.]

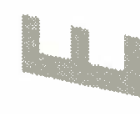




\section{BRÈVES MOII}

Pouvoir transformant des gènes homéotiques. Les gènes homéotiques - d'abord décrits chez la drosophile, où leur altération modific le schéma de développement de la mouche - sont caractérisés par la présence d'un domaine protéique de fixation à l'ADN, très conservé au cours de l'évolution, et qui porte le nom de "boîte homéo ". Chez la souris, une famille d'homéogènes appclés Hox a été identifiéc par criblage à l'aide des sondes correspondant aux gènes de drosophile. L'analyse de la régulation de l'expression de ces gènes au cours du développement de la souris a permis de suggérer que le sytème nerveux central des mammiferes pouvait être divisé en domaines dessinés par le prolil d'expression des gènes homéotiques [1]. De tels gènes, codant pour des facteurs transcriptionnels, impliqués dans le contrôle du développement, pouvaient être considérés comme des oncogènes potentiels. Le potenticl oncogénique du gène Hox-2.4 vient d'être confirmé par Aberdam D. de l'Institut Weizmann (Israël) [2]. Dans unc première séric d'expériences, la même équipe avait recherché des perturbations de gènes homéotiques au sein de tumeurs murines et identilié, dans des cellules leucémiques, un réarrangement du gène Hox-2. 4 induit par l'insertion d'unc séquence IAP (intracysternal $A$ particle). Ce réarrangement a pour conséquence l'expression d'un transcrit Hox-2.4 anormal dans des cellules qui n'expriment normalement pas le gène Hox-2.4 [3, 4]. Pour démontrer l'implication directe de cette séquence dans la transformation, les autcurs ont cloné le gène Hox-2.4 réarrangé (IAP-Hox-2.4) et l'ont introduit par transfection dans des fibroblates NIH 3T3. Transférés chez. la souris nude, les fibroblates exprimant le gène IAP-Hox-2.4 sont à l'origine de tumeurs plus ou moins agressives selon le point d'injection. Ainsi, le potenticl oncogénique de gènes homéotiques activés est-il démontré. Chez l'homme, l'analyse moléculaire d'une translocation $t$ (1; 19) dans des cellules pré-B d'une leu- cémic lymphoblastique aiguë provoque un réarrangement du gène homéotique $\mathrm{prl}\left(\mathrm{m} / \mathrm{s}, \mathrm{n}^{\circ} 5\right.$, vol. 6 , p. 489). Ainsi, les gènes homéotiques peuvent être ajoutés à la liste déjà longue des oncogènes potenticls.

[1. Tuggle CK, et al. Genes Dev $1990 ; 4: 180-9$.]

[2. Aberdam D, et al. Mol Cell Biol 1991; 11 : 554-7.]

[3. Blatt C, et al. EMBO J $1988 ; 7$ : 4283-90.]

[4. Kongsuwan K, el al. Nuclcic Acids Res 1989; 17: 1881-92.]

Inactivation par double recombinaison homologue des deux allèles du gène pim-1. Pour étudier, ex vivo, les conséquences phénotypiques de la non-expression d'un gène, des modèles cellulaires dérivés de souris transgéniques homozygotes dans lesquelles le gène à inactiver a été remplacé par le transgène peuvent être utilisés. La méthodologic récente, mais déjà classique, pour obtenir le remplacement des deux allèles d'un gène par une séquence exogène, repose sur la recombinaison homologue dans les cellules embryonnaires, entre l'un des allèles du gène à inactiver et le transgène. L'insertion par recombinaison homologue étant chez les mammiferes beaucoup moins fréquente que l'intégration au hasard, il est nécessaire de sélectionner et de reconnaître les cellules dans lesquelles elle s'est produite [1]. Pour ce faire, les transgènes introduits comportent souvent, outre les séquences homologues à cibler, des gènes de sélection, l'un permettant de trier les cellules ayant intégré le transgène et l'autre permettant d'éliminer les cellules ayant fait l'objet d'une intégration au hasard. Les cellules embryonnaires recombinées, introduites dans des blastocystes, vont participer à la constitution de chimères constituées de cellules normales et de cellules recombinées. Lorsque ce chimérisme atteint les cellules germinales, des descendants transgéniques dont toutes les cellules comportent le transgène et l'allèle normal peuvent être produits. Par croisement, des animaux homozygotes pour le transgène, n'ayant donc plus d'allèle normal, sont obtenus, à partir desquels des modèles cellulaires sont dérivés. Une stratégic plus directe, fondéc sur l'inactivation séquenticlle de l'un puis de l'autre allèle du gène ciblé vient d'être appliquéc au gène pim-1 par Hein tc R et al. du Netherlands Cancer Institute (Amsterdam). Pour ce faire, un premier transgène - comportant le gène de résistance à la néomycine (néo) placé au sein de la séquence pim-1 de telle sorte qu'il ne puisse fonctionner que lorsque le transgène est intégré à la place du gène endogène pirn-1 (pim-1/néo) - est utilisé. Dans les cellules résistantes à la néomycine, obtenues après transfert du gìne pim-1/néo, un second transgène - comportant, à la place du gène néo, le gène de résistance à l'hygromycine (pim-1/hyg) - est ensuite introduit. La sélection par l'hygromycine B permet alors de sélectionner les cellules dont le second allèle pim-1 a été remplacé par le transgène pim-1/hy'g. Les auteurs ont ainsi sélectionné des cellules ES pim-1/néo, pim-1/hy'g qui n'ont plus de gène pim-1 normal. I,'absence de la protéine Pim-1 dans les cellules ES où elle est normalement synthétiséc en abondance ne provoque pas de perturbations majeures de leur phénotype in vitro, permettant de formuler trois hypothèses : (1) il existerait, au cours du développement, une redondance de l'information, la fonction d'une protéine manquante pouvant être supplééc par une autre protéine ; (2) la protéine éliminée joucrait un rôle dans des conditions bien particulières auxquelles les cellules ES analysées in vitro n'auraient pas été soumises ; (3) la protéine Pim-1 interviendrait dans une cascade de régulations et de fonctions dont les anomalies ne seraient détectables qu'à un stade ultéricur, inaccessible à l'analyse in vitro.

[1. Lemarchandel $\mathrm{B}$, el al. médecine/sciences $1990 ; 6:$ 18-29.]

[2. Hein tc R, el al. Nature 1990 ; $348: 649-51$. 
Déficit partiel du transporteur de glucose GLUT-2 dans un modèle animal de diabète non insulinodépendant. Il existe plusicurs types de transporteurs de glucose, plus ou moins spécifiques de tissus et répondant, ou non, à l'insuline [1]. Dans le foie et les cellules $\beta$ des îlots de Langerhans du pancréas se trouve le transportcur GLUT-2, à haute constante de Michaelis (10 mM), qui assure un équilibre entre le glucose extra- et intracellulairc, le transport étant grossièrement proportionnel à la glycémie [2]. Les cellules $\beta$ langerhansiennes du rat Zucker, qui constitue un modèle de diabète non insulino-dépendant associć à une résistance à l'insuline et à une obésité, ont une densité extrêmement faible de molécules GLUT-2 [3], qui provoque probablement leur insuffisance de réponse sccrétoire insulinique au glucose. Il vient maintenant d'être démontré par des équipes suisse (Genève) et américaines (Indianapolis, IN, et Dallas, TX) que cette insuffisance d'expression de la protéine GLUT-2 préexistait chez le rat Zucker à toute hyperglycémie, et ne pouvait donc être attribuée à unc glucotoxicité comme d'autres anomalies de ce type de diabète [4]. Sans que le mécanisme du déficit partiel de GLUT-2 soit à cette heure connu, il pourrait s'agir là d'un désordre causal dans l'apparition du diabète. En effet, l'insulinosécrétion de la cellule $\beta$ langerhansienne est normalement contrôléc par la concentration de glucose extracellulaire, en équilibre grâce à GLUT-2 avec le glucose intracellulaire. Ce sucre, une fois entré dans la cellule, est métabolisé après avoir été phosphorylé par la glucokinase, ce qui conduit à la production d'énergie stockée sous forme d'ATP. L'ATP agit alors sur des canaux $\mathrm{K}+$ sensibles à ce trinucléotide, qui sont fermés, entraînant une dépolarisation membranairc et l'ouverture de canaux calciques dépendants du voltage [5]. La sécrétion d'insuline serait secondaire à l'augmentation de la concentration cytosolique du $\mathrm{Ca}^{2}+$. Une diminution de l'efficacité de la première étapc, l'entrée du glucose, peut donc expliquer la résistance au glucose de la cellule $\beta$ langerhansienne, l'insuffisance de la sécrétion d'insuline et l'hyperglycémie qui pourrait à son tour, par le mécanisme de la glucotoxicité évoqué plus haut, aboutir à la résistance à l'insuline [6].

[1. Assimacopoulos F, Jeanrenaud B médecine/sciences $1989 ; 5: 32-41$.]

[2. Thorens B, et al. Cell 1988 ; 55 : 281-90.]

[3. Johnson JH, et al. Science 1990 ; 250: 546-9.]

[4. Orci L, et al. Proc Natl Acad Sci USA 1990 ; 87 : 9953-7.]

[5. Lazdunski $M$. médecine/sciences $1990 ; 6: 279-85$.]

[6. Portha B. médecine/sciences 1991 ; 7: 212-25.]

Contrôle du sexe de l'enfant à naître. Du régime alimentaire précédant la fécondation aux tentatives diverses de fractionnement des spermatozoïdes, nombreuses sont les méthodes qui ont été rapportées comme étant capables de maîtriser le sexe des embryons, c'est-à-dire la nature du spermatozoïde fécondant. Ces derniers different en effet sclon qu'ils contiennent un chromosome $Y$ ou un chromosome $\mathrm{X}$, les premiers aboutissant à un embryon mâle et les seconds à un embryon femelle. Jusqu'à présent, cependant, aucune technique de fécondation avec contrôle du sexe n'a jamais donné significativement plus de $50 \%$ de succès... C'est sur des bases totalement nouvelles que reposent deux brevets récemment déposés par des sociétés américaines, l'une gouvernementale (le département du Commerce) et l'autre privée, Cytogam, de Chandler en Arizona. Le principe du tri de spermatozoïdes selon le sexe que décrivent ces brevets est leur marquage par un fluorochrome vital de type benzimide, puis leur séparation en trieur de cellules (cytométrie en flux, $m / s n^{\circ} 10$, vol. 6, p. 1000). Les spermatozoïdes $X$ sont légèrement plus fluorescents que les spermatozoïdes $Y$, et peuvent ainsi être séparés de ces dernicrs. Les premiers essais de ces méthodes chez l'animal (lapins et porcs) seraient extrêmement encourageants. Cytogam a aussi développé d'autres approches, complémentaires ou alternatives du tri en cytométric de flux. Il existe des "protéines associćes au sexe" à la surface des spermatozoïdes qui sont spécifiques des gamètes mâles ou femelles. Des anticorps appropriés ont été développés qui, ajoutés au sperme, inactivent la classe de spermatozoïdes contre laquelle ils sont dirigés, augmentant ainsi la chance d'obtenir, après fécondation, des embryons du sexe désiré. On peut même envisager d'immuniser des animaux femelles contre ces antigènes, créant ainsi des génitrices exclusives de descendants d'un sexe. Toutes ces nouveautés ont un grand intérêt dans le domaine de l'élevage. Appliquées à l'espèce humaine, elles soulèvent bien évidemment de très importantes questions éthiques qu'il ne faudra pas ćluder. Cette application à l'homme pourrait d'ailleurs être assez difficile, la différence entre les masses d'ADN des spermatozoïdes mâles et femelles étant moins importante que chez les animaux domestiques $(2,9 \%$ dans le premier cas, de 3 à $4,5 \%$ dans le second). Les familles à risque pour des maladies liées à au X (myopathie du Duchenne, hémophilie, par exemple) représentent de possibles indications d'une fécondation artificielle après tri de spermatozoïdes.

[Fox B, Joyce C. New Scientist 12 janvier 1991, 23.]

Un ADN monocaténaire détecté par microscopie électronique dans le cerveau d'animaux atteints de tremblante. La cause des encéphalopathies spongiformes représentées typiquement par la maladie de Creutzfeldt-Jakob chez l'homme et par la tremblante (scrapic) chez les animaux - reste incon- 


\section{口 BRÈVES}

nue. Devant l'impossibilité de mettre en évidence un ADN responsable, on a surtout discuté du pouvoir infectieux de protéines appelées prions (voir $\mathrm{m} / \mathrm{s} n^{\circ} 2$, vol. 7, p. 186). Un chercheur de Newcastle (RoyaumeUni) ouvre une voie intéressante par des méthodes morphologiques. Le travail de Narang [1] est fondé sur l'examen de 50 hamsters inoculés avec une suspension de cerveaux infectés qui provoque une tremblante clinique en deux à trois mois. Le cerveau de ces animaux présente des particules de tubulofilaments. Si on prépare l'ADN et qu'on l'examine en microscopie électronique après étalement sur des grilles recouvertes de collodion, on voit surtout, chez les malades comme chez les témoins, des molécules d'ADN double brin d'environ $16 \mathrm{~kb}$ qui ont tous les caractères de l'ADN mitochondrial. Mais en plus, chez les malades seuls, on peut voir des molécules d'ADN simple brin, dont on peut évaluer, d'après leur taille, le poids moléculaire à environ $0,5 \times 10^{6}$. Ni les témoins ni des animaux porteurs d'autres encéphalopathies n'ont montré unc telle image. Ces molécules résistent à l' ARNase, mais sont digérées par une combinaison de protéase et d'ADNase. Cette dernière enzyme seule est inefficace. On peut donc admettre que l'ADN monocaténaire mis ici en évidence est protégé par une couche de protéines. Si l'ensemble ADN-protéine est nécessaire à l'infectivité, on conçoit que cellc-ci puisse disparaître sous l'action d'agents qui détruisent ou inactivent les protéines, mais non sous celle des nucléases, qui n'auraient pas accès à l'ADN enfoui dans son manteau protéique. Ce travail est purement morphologique et la nature de l'ADN et de la protéine n'a pas été déterminée. Il peut cependant constituer un point de départ pour une attaque décisive sur la pathogénie de ces redoutables maladies.

[1. Narang HK. J Mol Biol 1990 ; 216: 469-73.]

\section{Empreinte parentale : expression exclusive du gène maternel du récepteur d'IGF-2}

Un ensemble d'observations remarquables effectuées ces dernières années [1, 2] a permis de mettre en évidence le fait que, chez la souris et sans doute chez les mammifères en général, le développement embryonnaire ne peut se dérouler normalement que si les cellules de l'embryon contiennent à la fois un génome d'origine maternelle et un génome d'origine paternelle. En d'autres termes, aucun embryon uniparental, parthénote (avec des chromosomes d'origine uniquement maternelle) ou androgénote (avec des chromosomes d'origine uniquement paternelle) ne se développe à terme.

On en a déduit que les génomes paternel et maternel subissent une " empreinte " entraînant une expression différentielle de certains gènes essentiels selon qu'ils sont portés par un chromosome d'origine paternelle ou maternelle. La nature de l'empreinte, le mode de régulation qu'elle entraîne restent cependant à découvrir. Il est donc essentiel d'isoler les gènes soumis à cette empreinte et de démontrer leur rôle dans le développement. S'il a été montré que certains transgènes paraissent se comporter, au moins approximativement, comme on l'attendrait de gènes soumis à l'empreinte parentale [3], aucun gène endogène subissant ce type de régulation n'avait été repéré jusqu'à présent. D'où l'intérêt de l'article de Barlow et al de Vienne (Autriche), Tübingen (Allemagne) et Nashville (TE, USA) paru dans le numéro de Nature du 3 janvier 1991 [4], décrivant la caractérisation d'un gène murin dont l'expression dépend de l'origine parentale du chromosome qui le porte. Les auteurs ont mis à profit l'existence de deux mutations $\mathrm{T}^{\text {hp }}$ et $\mathrm{t}^{\text {Lub2}}$, des délétions chevauchantes situées dans la même région du chromosome 17. Ces mutations entraînent la mort de l'embryon qui les porte, mais seulement si elles ont été transmises par la mère. Le comportement de ces mutations s'interprète aisément si l'on admet que le locus délété contient un gène qui ne s'exprime que s'il est porté par le chromosome d'origine maternelle et donc est soumis à unc cmpreinte génomique parentale. C'est exactement ce qu'orit trouvé les auteurs en combinant de manière élégante une approche génétique et moléculaire, ce qui a posteriori rend encore plus judicieux le nom qui avait été donné à ce locus: Tme pour $T$ associated maternel effect.

Ils ont d'abord observé qu'un gène, Tcp1, est délété dans les deux chromosomes $\mathrm{t}^{\text {Lub-2}}$ et $\mathrm{T}^{\text {hp }}$ et que son équivalent humain est très lié sur la carte génétique à trois autres gènes : ceux du plasminogène $(P l g)$, de la superoxyde dismutase mitochondriale (Sod-2) et du récepteur de l'IGF-2 (insulin-like growth factor 2) (IGF-2-r). La localisation de ces trois gènes a alors été effectuée sur le chromosome de souris et a permis d'établir les bornes de la délétion $\mathrm{t}^{\mathrm{Lub}-2}$ (la plus petite des deux délétions) définissant ainsi une région minimale où se trouve le locus Tme. Le résultat de cette analyse est indiqué sur la figure 1 et montre que les gènes $I G F-2-r, T c p 1$ et $\operatorname{Sod}-2$ sont délétés dans le chromosome $\mathrm{t}^{\text {Lub-2}}$, un gène supplémentaire Plg étant délété dans le chromosome

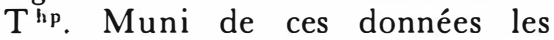
auteurs ont effectué les deux croisements réciproques $\mathrm{T}^{\mathrm{hp}} / \mathrm{x}$ 